\title{
Fatal meningococcal meningitis in a HIV-infected patient caused by serogroup C Neisseria meningitidis belonging to the non-hypervirulent clonal complex ST-60 (cc60)
}

\author{
Authors \\ Claudia Ferreira de \\ Andrade ${ }^{1}$ \\ Denise Cotrim da Cunha ${ }^{2}$ \\ Veronica Cavalcanti ${ }^{3}$ \\ Ivano de Filippis ${ }^{4}$ \\ ${ }^{1}$ Biologist; Laboratory \\ Technician, INCQS/ \\ FIOCRUZ, Rio de Janeiro, \\ Brazil. \\ ${ }^{2} \mathrm{MD}$; Physician, Hospital \\ Lourenço Jorge, Rio de \\ Janeiro, Brazil \\ ${ }^{3}$ Biologist; Clinical \\ Pathology Technician, \\ Hospital Lourenço Jorge, \\ Rio de Janeiro, Brazil \\ ${ }^{4}$ Post-doctorate; Senior \\ Technologist, INCQS/ \\ FIOCRUZ, Rio de Janeiro, \\ Brazil.
}

Submitted on: 2/18/2010

Approved on: 6/17/2010

Correspondence to:

Ivano de Filippis

Instituto Nacional de

Controle de Qualidade

em Saúde

Fundação Oswaldo Cruz

Av. Brasil, 4365,

Rio de Janeiro, Brazil

21045-900

Phone: +55-21-3865-5236

Fax: +55-21-2290-0915

ivano.defilippis@incqs.

fiocruz.br

We declare no conflict of interest.

\begin{abstract}
Meningococcal strains belonging to clonal complex cc60 are not associated with hypervirulent lineages and were never reported as causing disease in Latin American countries. This is the first report of a fatal meningitis case caused by a cc60 clonal complex meningococcus in Brazil. Despite the immune-compromised state of the patient, the fatal outcome here described shows the potential pathogenic behavior of strains belonging to this clonal complex and how compromised hosts can be susceptible to meningococcal infections even if the strain is not particularly invasive.
\end{abstract}

Keywords: meningitis, meningococcal; meningococcal infections; Neisseria meningitidis; serogroup C.

[Braz J Infect Dis 2011;15(2):178-180]@Elsevier Editora Ltda.

\section{INTRODUCTION}

Meningococcal disease $(\mathrm{MD})$ is a life-threatening illness that may affect susceptible patients and is presented in two clinical forms: meningitis and septicemia. Both forms lead to high fatality rates ranging from $5 \%$ in developed countries to $20 \%$ in developing countries. When not fatal, the disease may leave severe sequelae. The etiological agent of MD is the bacterium Neisseria meningitidis (meningococcus), a Gram-negative diplococcus harboring a polysaccharide capsule (PSC) which is the main responsible for the severity of the disease when it is released into the bloodstream during the infection process. The capsular polysaccharide of the meningococcus has different biochemical features which are used for typing the microorganism into 13 different serogroups according to their reactivity against specific antisera. Despite the great number of different serogroups, only 5 (A, B, C, W135 and Y) account for more than $90 \%$ of the strains producing disease worldwide. Serogrouping has been widely used in epidemiological studies to track epidemic strains and the PSC has been the main target of several effective vaccines against meningococci.

A number of proteins exposed on the meningococcal outer membrane have been used for typing strains giving an additional typing system dividing strains into serotypes and serosubtypes. Specific and consistent groups of serotypes and serosubtypes have been associated with diseases caused by certain serogroups. Also, a widely used molecular typing method called multilocus sequence typing (MLST) that indexes variation in multiple housekeeping genes, has been successfully applied to track hypervirulent strains during outbreaks. Under this molecular approach, a huge database of sequence types (ST) and serosubtypes has been developed and is hosted at the Oxford University, UK, http://neisseria. org/nm/typing/. ${ }^{1,2}$

The so-called clonal complexes (cc) identified by MLST studies, are the main unit for epidemiological analysis of meningococci. A sub-group of cc, the so-called hyperinvasive or hypervirulent lineages, has been responsible for most reported meningococcal disease globally during the latter half of the twentieth century. ${ }^{3}$ Hypervirulent cc are clearly diseaseassociated, but a great number of lineages has been only found in carriers such as the clonal complex 60 (cc60). Strains belonging to this $\mathrm{cc}$ have been isolated in Europe, USA and Canada from nasopharynx of healthy carriers and from sporadic invasive disease. To our knowledge, cc60 was never reported in Latin American countries. 


\section{CASE REPORT}

We report the case of a 33 year old female patient previously diagnosed with HIV and currently on antiviral therapy (AZT/3TC/lopinavir + ritonavir). She was admitted to a public hospital in Rio de Janeiro, Brazil, because of loss of consciousness with suspected bacterial meningitis, toxoplasmosis or cryptococcosis of the CNS. A xanthochromic turbid CSF was collected on the $2^{\text {nd }}$ day of admission and cultivated in chocolate agar with negative growth after $48 \mathrm{~h}$ of incubation in a $5 \% \mathrm{CO}_{2}$ atmosphere. Hemoculture, latex seroagglutination and Ziehl-Neelsen stain (AFB) also turned out negative. CSF white blood cell count was 25 cells with $80 \%$ monocytes and $20 \%$ PMN. Antibiotic therapy was initiated with ceftriaxone, sulfadiazine and pyrimethamine on the $3^{\text {rd }}$ day of admission. On the $4^{\text {th }}$ day the patient died after tracheal intubation and use of vasoactive amines.

A CSF aliquot collected on the $2^{\text {nd }}$ day was sent to our laboratory for investigation of bacterial meningitis pathogens Neisseria meningitidis (Nm), Haemophilus influenzae (Hi) and Streptococcus pneumoniae (Sp) by molecular methods. The CSF sample was firstly inoculated into Brain Heart Infusion chocolate agar (Difco) enriched with $10 \%$ defibrinated rabbit blood at $37^{\circ} \mathrm{C}$, but no growth was observed after $48 \mathrm{~h}$ in $5 \% \mathrm{CO}_{2}$ atmosphere. The CSF sample was then used for DNA extraction and purification with DNeasy Blood \& Tissue kit (Qiagen) and submitted to a multiplex-PCR approach using specific primers for detection of the three bacterial agents previously described. The targeted genomic regions were the outer membrane protein $n s p \mathrm{~A}$ gene for $\mathrm{Nm}$, the capsular gene bexA for $\mathrm{Hi}$ and the pneumolysin gene ply for Sp. The primers used for the specific PCR amplification were previously described for $n s p \mathrm{~A}^{4}{ }^{4}$ bex $\mathrm{A}^{5}$ and $p l y .{ }^{6}$ Agarose gel electrophoresis of PCR products, showed a unique $481 \mathrm{bp}$ band confirming the presence of meningococcal genomic DNA in the CSF sample received. CSF DNA sample was also analyzed aiming at genotype determination of the outer membrane protein genes porA, porB, fet $A$ and for MLST housekeeping genes analysis for sequence type (ST) and clonal complex (cc) characterization. Serogroup prediction was also performed with the DNA samples, using the SIAD-PCR previously described ${ }^{7}$ since latex agglutination test for serogroup determination was negative for serogroups $\mathrm{A}, \mathrm{B}, \mathrm{C}$ and W135/Y. For PCR amplification and DNA sequencing of the above mentioned genes, we followed the protocol described in the Nesisseria.org database at http://neisseria. org/nm/typing/. Sequencing results showed the following genotype: C:NT:P1.22,14-6,36-2: ST-7327 (cc60). After many attempts and protocol changes, we were not able to amplify por $B$ and fet $A$ genes. This was probably due to degradation of the genomic DNA extracted from the CSF sample received from the hospital.

\section{DISCUSSION}

Serogroups $\mathrm{B}$ and $\mathrm{C}$ meningococci, account for more than $90 \%$ of meningococcal cases in Brazil. Recent studies showed that the most frequent genotypes in Brazil are B:4,7:P1.19,15(P1.7,1):ST-33 (cc32) and C:2b:P1.3(P1.2):ST-103 (cc103), but other hyperinvasive $\mathrm{cc}$ also have been found among Brazilian isolates. ${ }^{8-12}$ These cc, also called hypervirulent, are part of a group of nine hyperinvasive lineages associated with most endemic and epidemic meningococcal disease of the latter half of the twentieth century. The distribution of such lineages varies within geographical regions. In Brazil, non-hypervirulent cc causing invasive disease is not frequent. According to the Neisseria.org database, $31.4 \%$ of the isolates characterized by MLST in Brazil $(n=271)$, belong to non-hypervirulent cc; however, none of the isolates was associated with cc60. Worldwide, the occurrence of cc60 strains is $1.9 \%$ according to the Neisseria. org database. To date, the total number of isolates reported by the database is 13,621 with only 260 strains belonging to $c c 60$. The association of a specific serogroup, serotype or subtype to cc60 as it is observed with hypervirulent $\mathrm{cc}$ is not clear. The strain isolated from the patient here described belongs to serogroup C, serotype not determined and subtype P1.22,14-6. Among the 260 cc60 strains reported worldwide, only 7 (2.7\%) were serogroup $\mathrm{C}$ and only one isolate $(0.4 \%)$ belonged to families 22 and 14 of PorA VR1 and VR2, respectively. Also, $57 \%$ of the cc60 isolates reported in the Neisseria. org database were recovered from carriers, while $36.5 \%$ from disease and other $6.5 \%$ from unknown source. The genetic heterogeneity found among cc60 strains has been more commonly observed within carrier isolates, while hypervirulent associated strains frequently show a clear link to specific serogroups and serosubtypes. It is noteworthy that although the meningococcal strain was isolated from an HIV positive patient, the organism showed a high virulence demonstrated by the short period of time elapsed from the onset of symptoms to the patient death. This aggressive pattern is often observed with hypervirulent associated lineages.

The first report of a meningococcus strain belonging to cc60 in Brazil, causing fatal meningitis even in an immune-compromised HIV patient, may be seen as a concerning issue. The fatal outcome of case here described shows the potential pathogenic behavior of strains belonging to cc60 clonal complex and how compromised hosts can be susceptible to meningococcal infections even if they are not particularly invasive.

Improvement of the public health surveillance system would be advisable to monitor the spread of this clone within Brazil and adjacent Latin American countries. 


\section{ACKNOWLEDGMENTS}

This study made use of the Neisseria multilocus sequence typing web site (http://pubmlst.org/neisseria/) developed by Man-Suen Chan and Keith Jolley and sited at the University of Oxford (curator, Keith Jolley) and the N. meningitidis PorA typing web site (http://neisseria.org/nm/typing/), developed by Keith Jolley (curators, Janet Suker and Ian Feavers, respectively). The authors thank the "Plataforma Genomica - Sequenciamento de DNA PDTIS-FIOCRUZ' for the sequencing reactions. This work was funded by INCQS/FIOCRUZ (MS), Brazil.

\section{REFERENCES}

1. Maiden MC, Bygraves JA, Feil E et al. Multilocus sequence typing: a portable approach to the identification of clones within populations of pathogenic microorganisms. Proc Natl Acad Sci U S A. 1998; 17;95(6):3140-5.

2. Urwin R, Russell JE, Thompson EA et al. Distribution of surface protein variants among hyperinvasive meningococci: implications for vaccine design. Infect Immun. 2004; 72(10):5955-62.

3. Caugant DA. Population genetics and molecular epidemiology of Neisseria meningitidis. Apmis 1998; 106:505-25.

4. de Filippis I, do Nascimento CR, Clementino MB et al. Rapid detection of Neisseria meningitidis in cerebrospinal fluid by one-step polymerase chain reaction of the nspA gene. Diagn Microbiol Infect Dis. 2005; 51(2):85-90.

5. Falla TJ, Crook DW, Brophy LN et al. PCR for capsular typing of Haemophilus influenzae. J Clin Microbiol. 1994; 32(10):2382-6.
6. Toikka P, Nikkari S, Ruuskanen O et al. Pneumolysin PCRbased diagnosis of invasive pneumococcal infection in children. J Clin Microbiol. 1999; 37(3):633-7.

7. Pollard AJ, Probe G, Trombley C et al. Evaluation of a diagnostic polymerase chain reaction assay for Neisseria meningitidis in North America and field experience during an outbreak. Arch Pathol Lab Med. 2002; 126(10):1209-15.

8. Baethgen LF, Weidlich L, Moraes C et al. Epidemiology of meningococcal disease in southern Brazil from 1995 to 2003, and molecular characterization of Neisseria meningitidis using multilocus sequence typing. Trop Med Int Health. 2008; 13(1):31-40.

9. de Filippis I, de Andrade CF, Silva L et al. PorA variable antigenic regions VR1, VR2, and VR3 of Neisseria meningitidis serogroups B and C isolated in Brazil from 1999 to 2004. Infect Immun. 2007; 75(7):3683-5.

10. de Filippis I, Vicente AC. Multilocus sequence typing and repetitive element-based polymerase chain reaction analysis of Neisseria meningitides isolates in Brazil reveal the emergence of 11 new sequence types genetically related to the ST-32 and ST-41/44 complexes and high prevalence of strains related to hypervirulent lineages. Diagn Microbiol Infect Dis. 2005; 53(3):161-7.

11. de Lemos AP, Yara TY, Gorla MC et al. Clonal distribution of invasive Neisseria meningitidis serogroup C strains circulating from 1976 to 2005 in greater São Paulo, Brazil. J Clin Microbiol. 2007; 45(4):1266-73.

12. Lemos AP, Brandão AP, Gorla MC et al. Phenotypic characterization of Neisseria meningitidis strains isolated from invasive disease in Brazil from 1990 to 2001. J Med Microbiol. 2006; 55(Pt 6):751-7. 